\title{
Accuracy of whole-genome sequence imputation using hybrid peeling in large pedigreed livestock populations
}

Roger Ros-Freixedes ${ }^{1,2^{*}}$, Andrew Whalen ${ }^{1}$, Ching-Yi Chen ${ }^{3}$, Gregor Gorjanc ${ }^{1}$, William O. Herring ${ }^{3}$, Alan J. Mileham ${ }^{4}$ and John M. Hickey ${ }^{1}$

\begin{abstract}
Background: The coupling of appropriate sequencing strategies and imputation methods is critical for assembling large whole-genome sequence datasets from livestock populations for research and breeding. In this paper, we describe and validate the coupling of a sequencing strategy with the imputation method hybrid peeling in real animal breeding settings.
\end{abstract}

Methods: We used data from four pig populations of different size (18,349 to 107,815 individuals) that were widely genotyped at densities between 15,000 and 75,000 markers genome-wide. Around 2\% of the individuals in each population were sequenced (most of them at $1 \times$ or $2 x$ and 37-92 individuals per population, totalling 284, at 15-30x). We imputed whole-genome sequence data with hybrid peeling. We evaluated the imputation accuracy by removing the sequence data of the 284 individuals with high coverage, using a leave-one-out design. We simulated data that mimicked the sequencing strategy used in the real populations to quantify the factors that affected the individualwise and variant-wise imputation accuracies using regression trees.

Results: Imputation accuracy was high for the majority of individuals in all four populations (median individual-wise dosage correlation: 0.97). Imputation accuracy was lower for individuals in the earliest generations of each population than for the rest, due to the lack of marker array data for themselves and their ancestors. The main factors that determined the individual-wise imputation accuracy were the genotyping status, the availability of marker array data for immediate ancestors, and the degree of connectedness to the rest of the population, but sequencing coverage of the relatives had no effect. The main factors that determined variant-wise imputation accuracy were the minor allele frequency and the number of individuals with sequencing coverage at each variant site. Results were validated with the empirical observations.

Conclusions: We demonstrate that the coupling of an appropriate sequencing strategy and hybrid peeling is a powerful strategy for generating whole-genome sequence data with high accuracy in large pedigreed populations where only a small fraction of individuals $(2 \%)$ had been sequenced, mostly at low coverage. This is a critical step for the successful implementation of whole-genome sequence data for genomic prediction and fine-mapping of causal variants.

*Correspondence: roger.ros@roslin.ed.ac.uk

${ }^{1}$ The Roslin Institute and Royal (Dick) School of Veterinary Studies, The University of Edinburgh, Easter Bush, Midlothian, Scotland, UK

Full list of author information is available at the end of the article

\section{Background}

Sequence data has the potential to empower the identification of causal variants that underlie quantitative traits or diseases [1-4], enhance livestock breeding [5-7], and increase the precision and scope of population genetic

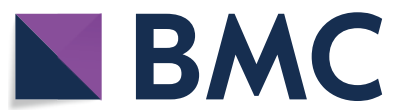

(c) The Author(s) 2020. This article is licensed under a Creative Commons Attribution 4.0 International License, which permits use, sharing, adaptation, distribution and reproduction in any medium or format, as long as you give appropriate credit to the original author(s) and the source, provide a link to the Creative Commons licence, and indicate if changes were made. The images or other third party material in this article are included in the article's Creative Commons licence, unless indicated otherwise in a credit line to the material. If material is not included in the article's Creative Commons licence and your intended use is not permitted by statutory regulation or exceeds the permitted use, you will need to obtain permission directly from the copyright holder. To view a copy of this licence, visit http://creativeco mmons.org/licenses/by/4.0/. The Creative Commons Public Domain Dedication waiver (http://creativecommons.org/publicdomain/ zero/1.0/) applies to the data made available in this article, unless otherwise stated in a credit line to the data. 
studies $[8,9]$. For sequence data to be used routinely in research and breeding, low-cost sequencing strategies must be deployed in order to assemble large datasets that capture most of the sequence diversity in a population and enable harnessing of its potential. One possible strategy is to sequence a subset of the individuals in a population at low coverage and then to perform imputation of whole-genome sequence data for the remaining individuals [10-12].

Such a strategy is likely to perform well in livestock breeding populations, in which individuals have a high degree of relatedness, allowing low-coverage sequence data to be pooled across individuals that share a haplotype and imputed to individuals who share that haplotype. Due to the implementation of genomic selection in livestock breeding populations, many individuals in breeding nucleus populations have already been genotyped with marker arrays. This genotype data can be used to identify the individuals that share haplotype segments and to select individuals for sequencing that will be more informative from an imputation perspective given a limited budget $[13,14]$.

We have recently proposed 'hybrid peeling' [15], a fast and accurate imputation method explicitly designed for jointly calling, phasing and imputing whole-genome sequence data in large and complex multi-generational pedigreed populations in which individuals can be sequenced at variable coverage or not sequenced at all. Hybrid peeling is a two-step process. In the first step, multi-locus iterative peeling is performed to estimate the segregation probabilities for a subset of segregating sites (e.g., the markers on a genotyping array). In the second step, the segregation probabilities are used to perform fast single-locus iterative peeling on every segregating site discovered in the genome. This two-step process allows the computationally demanding multi-locus peeling step to be performed on only a subset of the variants, while still leveraging linkage information for the remaining variants.

These properties make hybrid peeling a very appealing imputation method for the cost-effective generation of whole-genome sequence data for large pedigreed populations that have already been extensively genotyped using marker arrays and in which a small proportion of the individuals have been sequenced with variable coverage. In the situations described, the sequence data will be sparsely distributed across the pedigree and there may be great variability in the amount of data to which each individual is exposed. Understanding which factors affect individual-wise and variant-wise imputation accuracy and how their effects are mediated is important for determining how this sequencing strategy, together with hybrid peeling, performs in real settings that are common in animal breeding and for enabling accuracy-aware quality control of the imputed data before downstream analyses. Such knowledge could be used in the future to design cost-effective routine whole-genome sequencing strategies.

The objectives of this study were to: (i) demonstrate whether whole-genome sequence data could be imputed with high accuracy in a variety of pig pedigrees when small subsets of individuals are sequenced, mostly at low coverage; (ii) quantify the factors that determine the individual-wise and variant-wise imputation accuracy; and (iii) quantify the impact of data misassignment and pedigree errors on imputation accuracy. Our results showed that high overall imputation accuracies can be achieved for whole-genome sequence data in large pedigreed populations using hybrid peeling provided that the individuals are connected to a sufficient number of informative relatives with marker array or sequence data. Our results have implications for the practical implementation of sequencing and imputation strategies.

\section{Methods}

We structured the study in three tests. In Test 1 , we evaluated the imputation accuracy of hybrid peeling in four populations of different sizes. In Test 2, we used regression trees and simulated data based on three real pedigrees to quantify which factors determined the individual-wise and variant-wise imputation accuracy of hybrid peeling. Then, we used the observations in the real data to validate the findings and to predict individuals with low imputation accuracy. In Test 3, we evaluated the potential impact that data misassignment and pedigree errors could have on the imputation accuracy. In the following sections, we first describe how the data was generated and then how the different tests were performed.

\section{Real data}

\section{Populations and sequencing strategy}

We performed whole-genome sequencing of 4427 individuals from four commercial pig breeding lines (Genus PIC, Hendersonville, TN) using a total coverage of approximately $18,514 \times$. To account for a range of population sizes, the number of individuals in each population was 18,349 (20 k), 34,425 (35 k), 68,777 (70 k), or 107,815 $(110 \mathrm{k})$. Approximately $2 \%(1.7-2.5 \%)$ of the individuals in each population were sequenced, mostly at low coverage. The number of individuals sequenced and the coverage at which they were sequenced are summarized for each population in Table 1.

We selected the individuals and the coverage at which they were sequenced using a three-step strategy: (1) first, we selected sires and dams that contributed most genotyped progeny in the pedigree (referred to as top sires 
Table 1 Distribution of sequencing coverages by population

\begin{tabular}{lllllll}
\hline Population & \multicolumn{2}{c}{ Individuals sequenced } & \multicolumn{2}{c}{ Individuals sequenced by coverage } & \multicolumn{2}{c}{ Total coverage } \\
\cline { 2 - 6 } & & $\mathbf{1 x}$ & $\mathbf{2 x}$ & $\mathbf{5 x}$ & $\mathbf{1 5 - 3 0 x}$ & \\
\hline $20 \mathrm{k}$ & 445 & 217 & 176 & 15 & 37 & $1852 \mathrm{x}$ \\
$35 \mathrm{k}$ & 760 & 394 & 274 & 27 & 65 & $3192 \mathrm{x}$ \\
$70 \mathrm{k}$ & 1366 & 685 & 545 & 44 & 92 & $5280 \mathrm{x}$ \\
$110 \mathrm{k}$ & 1856 & 1044 & 649 & 73 & 90 & $8190 \mathrm{x}$ \\
\hline
\end{tabular}

and dams') to be respectively sequenced at $2 \times$ and $1 \times$; (2) then, we used AlphaSeqOpt part 1 [13] to identify the individuals whose haplotypes represented the greatest proportion of the population haplotypes (referred to as 'focal individuals') and to determine an optimal level of sequencing coverage between $0 \times$ and $30 \times$ for these individuals and their immediate ancestors (i.e., parents and grandparents) under a total cost constraint; and (3) finally, we used the AlphaSeqOpt part 2 [14] to identify individuals that carried haplotypes whith a low cumulative coverage (i.e., lower than, $10 \times$ ) and distributed $1 \times$ sequencing amongst those individuals so that the cumulative coverage on the haplotypes could be increased (i.e., at or above $10 \times$ ). AlphaSeqOpt used haplotypes inferred from marker array genotypes (GGP-Porcine HD BeadChip; GeneSeek, Lincoln, NE), which were phased with AlphaPhase [16] and imputed with AlphaImpute [17]. The sequencing resources were split so that approximately $30 \%$ of the sequencing resources were used for sequencing the top sires at $2 \times, 15 \%$ for the top dams at $1 \times, 25 \%$ for the focal individuals and their immediate ancestors at variable coverage [13], and the remaining $30 \%$ for individuals that carried under-sequenced haplotypes at $1 \times$ [14]. In step 2, we identified 284 individuals across the four populations who were sequenced at high coverage $(15 \times$ or $30 \times)$. Many of these individuals sequenced at high coverage belonged to early generations of the pedigree of each population. The rest of the sequenced individuals were sequenced at low coverage $(1 \times, 2 \times$ or $5 \times)$.

We sorted the pedigrees of each population so that parents appeared before their progeny. Thus, relative position in the pedigree was used as a proxy for the generation to which an individual belonged.

\section{Sequencing and data processing}

Tissue samples were collected from ear punches or tail clippings. Genomic DNA was extracted using Qiagen DNeasy 96 Blood \& Tissue kits (Qiagen Ltd., Mississauga, ON, Canada). Paired-end library preparation was conducted using the TruSeq DNA PCR-free protocol (Illumina, San Diego, CA). Libraries for sequencing at low coverage $(1 \times$ to $5 \times)$ were produced with an average insert size of 350 base pairs and sequenced on a HiSeq 4000 instrument (Illumina, San Diego, CA). Libraries for sequencing at high coverage $(15 \times$ or $30 \times)$ were produced with an average insert size of 550 base pairs and sequenced on a HiSeq X instrument (Illumina, San Diego, CA). All libraries were sequenced at Edinburgh Genomics (Edinburgh Genomics, University of Edinburgh, Edinburgh, UK). Most pigs were also genotyped either at low density (LD; 15,000 markers) using the GGP-Porcine LD BeadChip (GeneSeek, Lincoln, NE) or at high density (HD; 75,000 markers) using the GGPPorcine HD BeadChip (GeneSeek, Lincoln, NE).

DNA sequence reads were pre-processed using Trimmomatic [18] to remove adapter sequences from the reads. The reads were then aligned to the reference genome Sscrofa11.1 (GenBank accession: GCA_000003025.6; [19]) using the BWA-MEM algorithm [20]. Duplicates were marked with Picard (http:// broadinstitute.github.io/picard). Single nucleotide polymorphisms (SNPs) were identified with the variant caller GATK HaplotypeCaller (GATK 3.8.0; [21, 22]) using default settings. Between 20 and 30 million SNPs were discovered in each population.

To avoid biases towards the reference allele introduced by GATK when applied on low-coverage sequence data, we extracted the read counts supporting each allele at each variant site with a pile-up function using the pipeline described in [23]. This pipeline uses the tool pysam (version 0.13.0; https://github.com/pysam-developers/ pysam), which is a wrapper around htslib and the samtools package [24]. We extracted the read counts for all biallelic SNP positions, after filtering out variants with a mean coverage 3 times greater than the average realized coverage (considered as indicative of potential repetitive regions) with VCFtools [25].

We performed additional quality control on the pedigree by determining the number of Mendelian inconsistencies (percentage of opposing homozygous) between each parent-progeny pair. We applied the following criteria: (1) we removed marker array or sequence data of an individual, when the genotype data was incompatible 
with that of all its available parents and progeny (this was done because it could indicate data misassignment for that individual); (2) we removed parent-progeny pedigree links when the genotype data available was incompatible for only a pair of individuals but not for their other parents and progeny; and (3) we created a dummy parent with no genotype data when the genotype data of a group of littermates was incompatible with one of its parents but both the parent and the littermates were not incompatible with the rest of their parents and progeny (this was done to preserve the full-sib relationship between those individuals).

\section{Simulated data}

In order to test the factors that influenced imputation accuracy, we simulated genetic data for three populations of different sizes: 15,187 (15 k), 29,974 (30 k), and 64,598 ( $65 \mathrm{k}$ ) individuals. The pedigrees of these populations were a subset of the real pedigrees of the $20 \mathrm{k}, 35 \mathrm{k}$, and $110 \mathrm{k}$ populations used for the analyses of real data. As in the analyses of real data, the pedigrees were sorted so that parents appeared before their progeny. Genomic data for each population were simulated using the software AlphaSim [26]. Each simulation was repeated twice and results were averaged across repetitions. Below, we present only a brief description of the simulation strategy. The full details of the simulation are described in a companion paper [27].

Genomic data were simulated for 20 chromosomes, each $100 \mathrm{cM}$ long. In total, 150,000 SNPs per chromosome (3 million SNPs genome-wide) were simulated in order to represent whole-genome sequence. A subset of 3000 SNPs per chromosome (60,000 SNPs genomewide) was used as a high-density marker array (HD). A smaller subset of 300 SNPs per chromosome (6000 SNPs genome-wide) nested within the HD marker array was used as a low-density marker array (LD). Each individual was assigned HD or LD marker array data based on the density at which they were genotyped in real data. The sequence read counts for each individual and SNP were simulated by sampling sequence reads using a Poissongamma model that gave variable sequenceability at each SNP and variable number of reads for each individual at each SNP $[10,28]$.

The individuals to be sequenced and their sequencing coverage were selected using a combination of pedigree- and haplotype-based methods that mimicked the sequencing strategy that was used for the real data. The total level of investment for sequencing was equivalent to the cost of sequencing $2 \%$ of the population at $2 \times$, and thus resulted in a similar number of sequenced individuals as in the real data.

\section{Imputation using hybrid peeling}

Imputation was performed in each population separately using hybrid peeling, as implemented in AlphaPeel [15], with the default settings. Hybrid peeling extends the methods of Kerr and Kinghorn [29] for single-locus iterative peeling and of Meuwissen and Goddard [30] for multi-locus iterative peeling to efficiently call, phase and impute whole-genome sequence data in complex multigenerational pedigrees. Multi-locus iterative peeling was performed on all available marker array data to estimate the segregation probabilities for each individual. The individuals genotyped with LD marker arrays were not imputed to HD prior to this step. The segregation probabilities were used for segregation-aware single-locus iterative peeling for the variant sites genome-wide.

\section{Imputation accuracy tests}

Test 1: Imputation accuracy in populations of different size The imputation accuracy in the real data was estimated using a leave-one-out design. In each leave-one-out round, hybrid peeling was performed after removing the sequence data of one of the 284 individuals that were sequenced at high coverage (either 15 or $30 \times$ ) in the four populations. We used the genotypes imputed for these individuals using the full data as the true genotypes. To reduce computational requirements, accuracy was assessed on a random subset of 50,000 non-consecutive SNPs from chromosome 5, which included all the markers from the arrays that map to this chromosome $(\sim 3000)$. Tests in other chromosomes gave similar results.

We measured individual-wise and variant-wise imputation accuracy with the correlation between the true genotypes and imputed dosages. The dosage correlation was calculated after correcting for minor allele frequency (MAF), as recommended by Calus et al. [31]. To facilitate comparison with other studies that report the uncorrected (raw) allele dosage correlations, in the context of this study we found that MAF-corrected correlations of $0.75,0.80,0.85,0.90$, and 0.95 were respectively equivalent to the raw correlations of $0.89,0.91,0.93,0.96$, and 0.98 . For the variant-wise imputation accuracy, we excluded the individuals that had the lowest imputation accuracy, predicted as described in Test 2 .

Test 2: Factors that affect individual-wise and variant-wise imputation accuracy

We assessed the factors that influenced imputation accuracy in the simulated data. We used simulated data to provide a much larger sample size where the true genotypes were known. We ran single-locus peeling on a random subset of 5000 non-consecutive SNPs taken from across three chromosomes to reduce 
computational requirements, although the full set of 20 chromosomes were simulated to represent realistic genetic architecture and haplotype diversity for the haplotype-based method AlphaSeqOpt. We assessed the factors that influenced imputation accuracy by building regression trees. The regression trees were built using the data from 219,518 simulated individuals and 30,000 variants (5000 variants from each population and replicate). The regression trees were built using the 'rpart' $\mathrm{R}$ package [32], allowing partitions that increased the $\mathrm{R}^{2}$ of the model by 0.005 at each step. Consecutive binary partitions based on the same variable were considered as multi-part.

The regression tree for the individual-wise imputation accuracy was based on the amount of information that was available for the individual itself and its close relatives (4 relationship levels: grandparents, parents, progeny, and grandprogeny). The factors included: (i) population size; (ii) marker array density of the individual (3 genotyping statuses: not genotyped, genotyped at LD, or genotyped at HD); (iii) number of close relatives that were genotyped at each genotyping density (12 variables; 4 relationship levels and 3 genotyping statuses); (iv) number of close relatives that were sequenced and their cumulative sequencing coverage ( 8 variables; 2 variables for each of the 4 relationship levels); and (v) connectedness to the population, which was measured as the sum of coefficients of relationship between an individual and the rest of individuals in the pedigree.

We tested the predictive capacity of the partitioning factors for identifying individuals with low imputation accuracy, defined as those below 0.95. For that purpose, we performed a tenfold cross-validation on the simulated data. We validated the results of the regression trees in the real data against the imputation accuracy observed in the 284 high-coverage individuals. For the analysis of the variant-wise imputation accuracy we used only the individuals that were predicted to have imputation accuracy above 0.95 based on the partitioning factors of the regression tree. To further assess which factors affected the individual-wise imputation accuracy in the real data, we fitted a linear model predicting imputation accuracy against each of the factors used for the regression tree.

The factors in the regression tree for the variant-wise imputation accuracy included: (i) population size; (ii) MAF; (iii) relative position of the variant within a chromosome; (iv) distance of a variant to the nearest variant from the marker array (this distance was 0 if that variant was present on the marker array); (v) cumulative sequencing coverage across individuals at that variant site; and (vi) number of individuals with at least one sequencing read covering that variant site.
Test 3: Impact of data misassignment and pedigree errors We tested the impact that data misassignment and pedigree errors could have on the imputation results by introducing deliberate errors to the real data. We considered three types of errors: sequence data misassignment, marker array data misassignment, and pedigree errors. For each type of error, we created 284 scenarios, in which we altered the data of each of the individuals that were sequenced at high coverage in each population, one at a time. The three types of errors were defined as follows, to represent some worst-case scenarios:

\section{Sequence data misassignment}

We replaced the sequence data of the target individual by that of a random individual from the same population that had been sequenced at high coverage.

\section{Marker array data misassignment}

We replaced the marker array data of the target individual by that of a random individual from the same population that had been genotyped at HD, regardless of its own genotyping status or density.

\section{Pedigree errors}

We assigned random progeny from one of the individuals sequenced at high coverage from the same population to the target individual.

The impact of the data misassignment and pedigree errors on imputation accuracy was measured as the correlation between the allele dosages using the correct data and the erroneous data. The impact of these errors was assessed on the target individual where the error was introduced but also on its grandparents, parents, progeny, and grandprogeny to evaluate how the errors could propagate to relatives of the target individual. In the case of the pedigree errors, we also assessed the impact of the pedigree error on the misassigned progeny and grandprogeny. As a control, we also assessed the allele dosage correlation on the target individual and its relatives when the data of the target individual was removed, as done in Test 1.

\section{Results \\ Individual-wise imputation accuracy in populations of different size}

The imputation accuracy in the real data was high for most of the tested individuals. The average individualwise dosage correlation was 0.94 but there was substantial variation with an asymmetrical distribution (median: 0.97; $\min : 0.11$; $\max$ : 1 ; interquartile range: 0.94-0.98). Many of the individuals in the earliest generations of the pedigree (some of the 106 individuals located in the first $20 \%$ of the pedigree) had a lower 
imputation accuracy than individuals in the remainder of pedigree. This pattern was observed for all four populations. Figure 1 shows the imputation accuracy plotted against relative position in the pedigree, the marker array density of the individual, or size of the population to which they belonged. The imputation accuracy of the individuals in later generations (the 178 individuals after the first $20 \%$ of the pedigree) was higher than that of individuals in the earliest generations, with an average dosage correlation of 0.97 and with much lower variability (median: 0.98; min: 0.69; max: 1 ; interquartile range: $0.96-0.99$ ).

The marker array density of the individuals was confounded with the number of ancestors that were genotyped with marker arrays. The non-genotyped individuals $(\mathrm{n}=19)$ and approximately half of the individuals genotyped at HD ( $n=87$ out of 157 ) belonged to early generations of the pedigree (Fig. 1a), which reduced their chances of having ancestors with genotype data and penalized the imputation accuracy for these two groups of individuals (Fig. 1b). On the contrary, most individuals genotyped at LD belonged to later generations $(n=91$ out of 108), ensuring that there was enough data for their ancestors to enable high imputation accuracies for the LD individuals. The average imputation accuracy correlation was 0.81 for the non-genotyped individuals, 0.94 for the HD individuals, and 0.96 for the LD individuals. The average imputation accuracy for the HD individuals in the earliest generations was lower (0.91) than for the HD individuals in later generations (0.97). For individuals in the later generations, there were no significant differences between marker array densities and the average imputation accuracy of both the HD and LD individuals was 0.97 .
There was no clear trend that population size affected imputation accuracy (Fig. 1c), especially for individuals in the later generations. The population with $35 \mathrm{k}$ individuals had higher imputation accuracy than the other three populations but this was more likely due to populationspecific characteristics, related to unbalanced distributions of the tested individuals across generations and genotyping statuses or potentially to pedigree structure, rather than population size. The $35 \mathrm{k}$ population had only 5 out of 65 high-coverage individuals in the first $20 \%$ of the pedigree, compared to a much greater proportion in the other populations (from 15 out of 37 in the $15 \mathrm{k}$ population to 56 out of 92 in the $65 \mathrm{k}$ population).

\section{Factors that affect individual-wise imputation accuracy}

The main factors that determined individual-wise imputation accuracy were whether the individual itself was genotyped with a marker array, the number of close relatives of that individual that were genotyped with a marker array (primarily parents and grandparents), and the connectedness of that individual to the rest of the population. The number of close relatives of an individual that were sequenced was a significant factor for the imputation accuracy of the 284 tested individuals in a linear model, but only the number of sequenced parents or progeny were influential partitioning factors in the regression trees based on the simulated data. The sequencing coverage of the relatives were not influential partitioning factors in the regression trees. The results were consistent between simulated and real data.

The regression tree for the factors that affect individual-wise imputation accuracy in the simulated data is shown in Fig. 2a. The first partitioning factor was the availability of marker array data of the grandparents. On
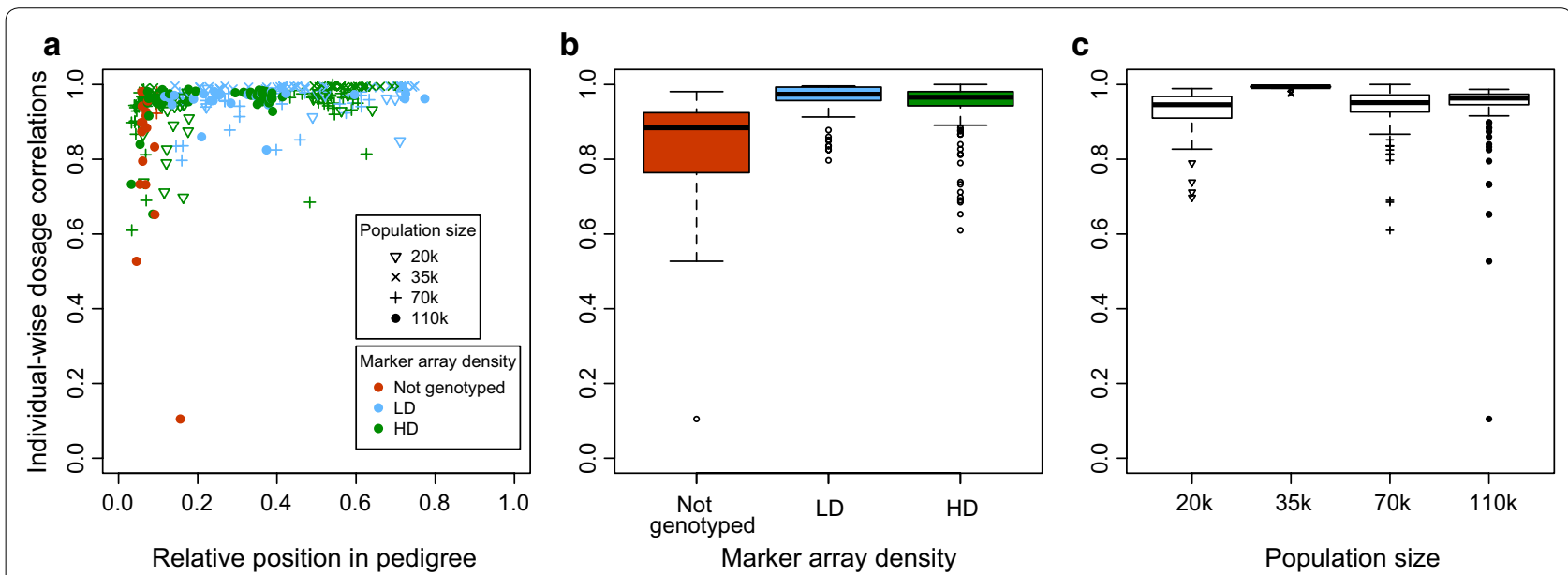

Fig. 1 Individual-wise dosage correlation in real data with respect to a relative position of the tested individuals within a pedigree $\mathbf{b}$ genotyping marker array density, and c population size 


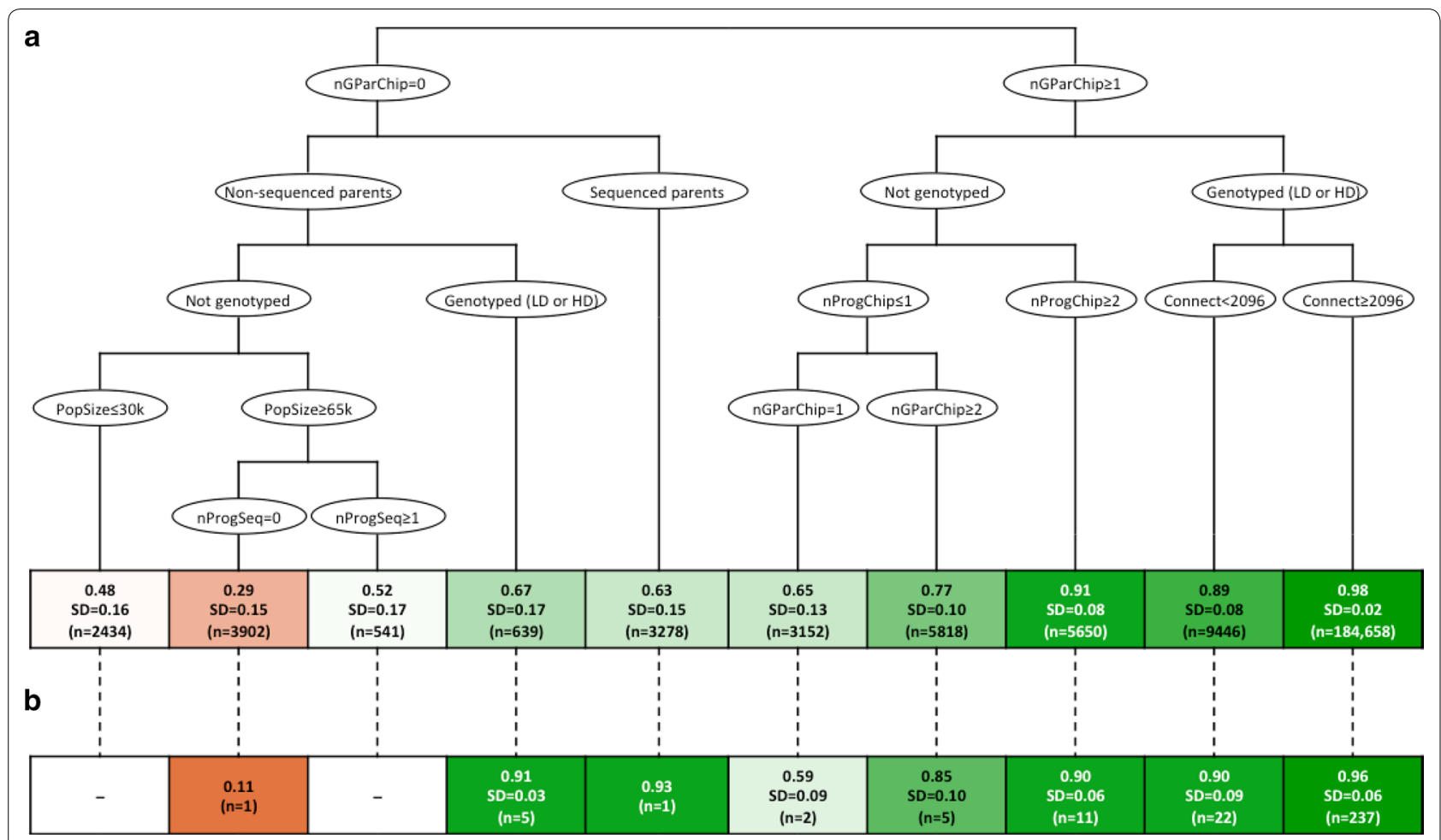

Fig. 2 Regression tree of the factors that affected individual-wise dosage correlation in $\mathbf{a}$ simulated data and $\mathbf{b}$ comparison to real data. Variables include genotyping status, number of grandparents genotyped with marker array (nGParChip), number of progeny genotyped with marker array (nProgChip), number of sequenced progeny (nProgSeq), connectedness to the rest of the population (Connect), and population size (PopSize)

average, individuals without genotyped grandparents had a much lower imputation accuracy $(0.47, \mathrm{n}=10,794)$ than individuals with at least one genotyped grandparent $(0.96, \mathrm{n}=208,724)$. For individuals without genotyped grandparents, other sources of information from the ancestors, such as availability of any sequenced parents, increased their imputation accuracy from $0.40(n=7516)$ to $0.63(\mathrm{n}=3278)$.

After these initial partitions, the next partitioning factor was whether or not the individual itself was genotyped with a marker array, regardless of marker array density. For non-genotyped individuals, having some genotyped or sequenced progeny and grandprogeny improved their imputation accuracy. For genotyped individuals, regardless of the genotyping density, connectedness to the rest of the population was the main factor that determined imputation accuracy, with the dosage correlation increasing with connectedness from 0.89 $(\mathrm{n}=9446)$ to $0.98(\mathrm{n}=184,658)$. The imputation accuracy observed in the real data was consistent with the partitions of the regression tree based on the simulated data (Fig. 2b).

These partitioning factors predicted the simulated individuals with low imputation accuracy (lower than 0.95 ) with a sensitivity of 0.66 and a specificity of 0.95 . In the real data, the correlation between the predicted and observed individual-wise imputation accuracy was 0.55 and the partitioning factors predicted the individuals with low imputation accuracy with a sensitivity of 0.42 and a specificity of 0.95 . These partitioning factors were sensitive for predicting the individuals with the lowest imputation accuracy but their sensitivity decreased for predicting individuals with imputation accuracy closer to the desired level of 0.95 (Table 2). In total, 237 individuals were predicted to have imputation accuracy higher than 0.95 and were later used for the analyses of variant-wise imputation accuracy.

The analysis of the factors that affected the individualwise imputation accuracy observed in the real data with a linear model largely supported the results of the regression trees. Table 3 summarises the factors that were significantly associated with individual-wise imputation accuracy. The significant factors included the number of genotyped ancestors (at HD; $p \leq 0.016$ ) but not the number of genotyped descendants $(p=0.062-0.996)$, and the number of sequenced relatives $(p \leq 0.016)$ but generally not their cumulative sequencing coverage $(p=0.044-$ 0.456 ). The factors that referred to the amount of information available for the individuals themselves were also significant, including both their genotyping status 
Table 2 Prediction of individuals with low imputation accuracy (below 0.95) using the partitioning factors from the regression tree

\begin{tabular}{llllllll}
\hline Data & Sensitivity & Specificity & \multicolumn{6}{l}{ Sensitivity by observed imputation accuracy } \\
\cline { 5 - 8 } & & & $\mathbf{0 - 0 . 5}$ & $\mathbf{0 . 5 - 0 . 7 5}$ & $\mathbf{0 . 7 5 - 0 . 8 5}$ & $\mathbf{0 . 8 5 - 0 . 9}$ & $\mathbf{0 . 9 - 0 . 9 5}$ \\
\hline Simulated & 0.66 & 0.97 & 1.00 & 0.99 & 0.94 & 0.68 & 0.27 \\
Real & 0.42 & 0.95 & 1.00 & 0.58 & 0.31 & 0.61 & 0.29 \\
\hline
\end{tabular}

Table 3 Factors that affect individual-wise imputation accuracy on the real data

\begin{tabular}{lr}
\hline Factor & $p$-value \\
\hline Population size & $<0.001$ \\
Individual data & \\
Genotyping status & $<0.001$ \\
Connectedness to the rest of population & 0.031 \\
Number of relatives genotyped with marker array & \\
Grandparents at LD & 0.707 \\
Grandparents at HD & 0.016 \\
Parents at LD & 0.059 \\
Parents at HD & $<0.001$ \\
Progeny at LD & 0.062 \\
Progeny at HD & 0.553 \\
Grandprogeny at LD & 0.926 \\
Grandprogeny at HD & 0.996 \\
Number of relatives sequenced & \\
Grandparents & 0.003 \\
Parents & $<0.001$ \\
Progeny & 0.002 \\
Grandprogeny & 0.016 \\
Cumulative sequencing coverage of relatives & \\
Grandparents & 0.456 \\
Parents & 0.245 \\
Progeny & 0.100 \\
Grandprogeny & 0.044 \\
\hline LD &
\end{tabular}

$L D$ low density, $H D$ high density

$(p \leq 0.001)$ and their connectedness to the rest of the population $(p=0.031)$. However, the marker array density was confounded with the generation to which the individuals belonged and, therefore, with the number of ancestors that were genotyped with marker arrays (Fig. 1). Population size was also a significant factor $(p \leq 0.001)$, but likely confounded with population-specific factors (Fig. 1).

\section{Variant-wise imputation accuracy}

The variant-wise imputation accuracy was also high. After removing the individuals with a low predicted imputation accuracy, the average variant-wise dosage correlation was 0.91 (median: 0.98; min: -0.37 ; max: 1 ; interquartile range: 0.94-0.99). We removed the individuals with a low predicted imputation accuracy to provide estimates of variant-wise imputation accuracy for the data that would pass such an initial quality control before any additional filtering steps or downstream analyses. Although removing such individuals resulted in slightly higher variant-wise imputation accuracy estimates than using all data ( 0.88 and 0.91 before and after filtering, respectively; correlation: 0.93 ), the results did not change.

Variant-wise imputation accuracy was lower for lowfrequency variants, compared to more common variants. Figure 3 shows the distribution of the imputation accuracy for variants across the MAF spectrum. The mean imputation accuracy increased from 0.60 for $\mathrm{MAF} \leq 0.001$ to 0.95 for $\mathrm{MAF} \geq 0.2$, but the median imputation accuracy was $>0.98$ for MAF $\geq 0.005$.

\section{Factors that affect variant-wise imputation accuracy}

The main factors that determined the variant-wise imputation accuracy were the MAF of the variants and the number of sequenced individuals at the variant site. Whether a marker was present in the marker array or not and the distance of a variant to the nearest variant from the marker array were not influential partitioning factors in the regression trees. The results were consistent between simulated and real data.

The regression tree for the factors that affect variantwise imputation accuracy on the simulated data is shown in Fig. 4a. The first factor that determined variant-wise imputation accuracy was MAF. The imputation accuracy was limited for very rare variants: 0.31 for MAF below $0.001(\mathrm{n}=395), 0.57$ for MAF between 0.001 and $0.005(\mathrm{n}=810), 0.85$ for MAF between 0.005 and 0.023 $(n=1810)$, and 0.97 for MAF above $0.023(n=26,268)$. The other partition factor was the number of individuals that had at least one sequencing read that covered a given position. The imputation accuracy observed in the real data within each partition of the regression tree followed the same trends as for the simulated data, but ranged from $0.55(n=7642)$ to $0.95(n=91,236)$ and were greater than those from the simulated data at low MAF (Fig. 4b). 


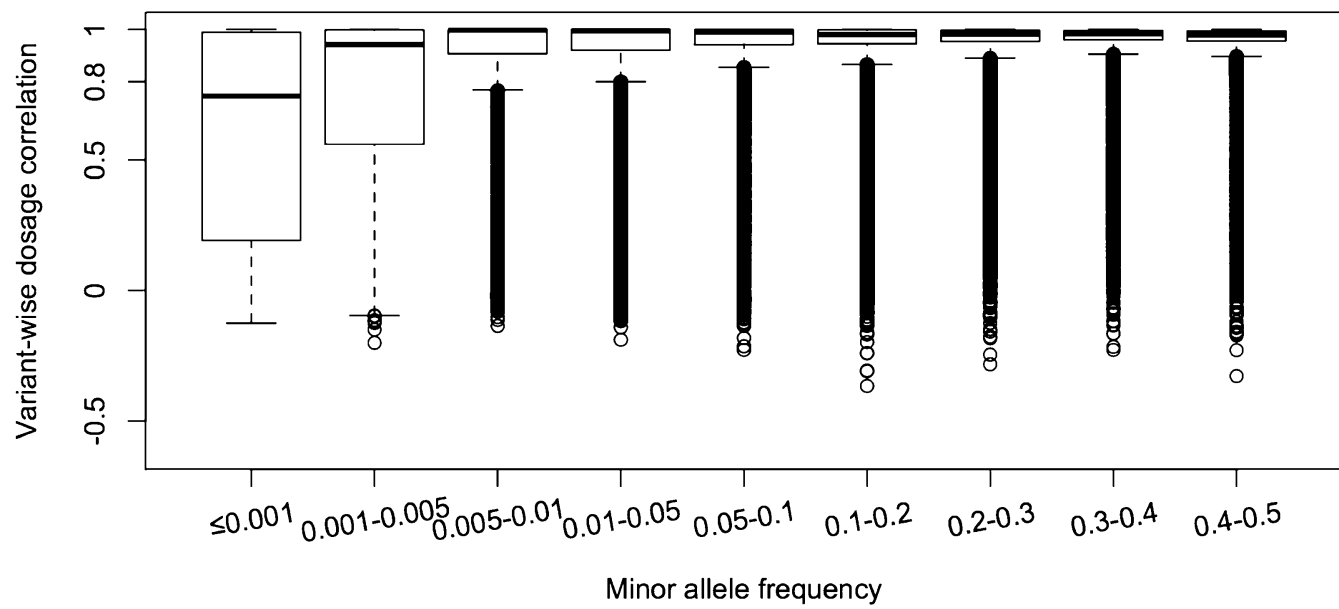

Fig. 3 Variant-wise dosage correlation in real data with respect to minor allele frequency. Results are after removing individuals with low predicted imputation accuracy

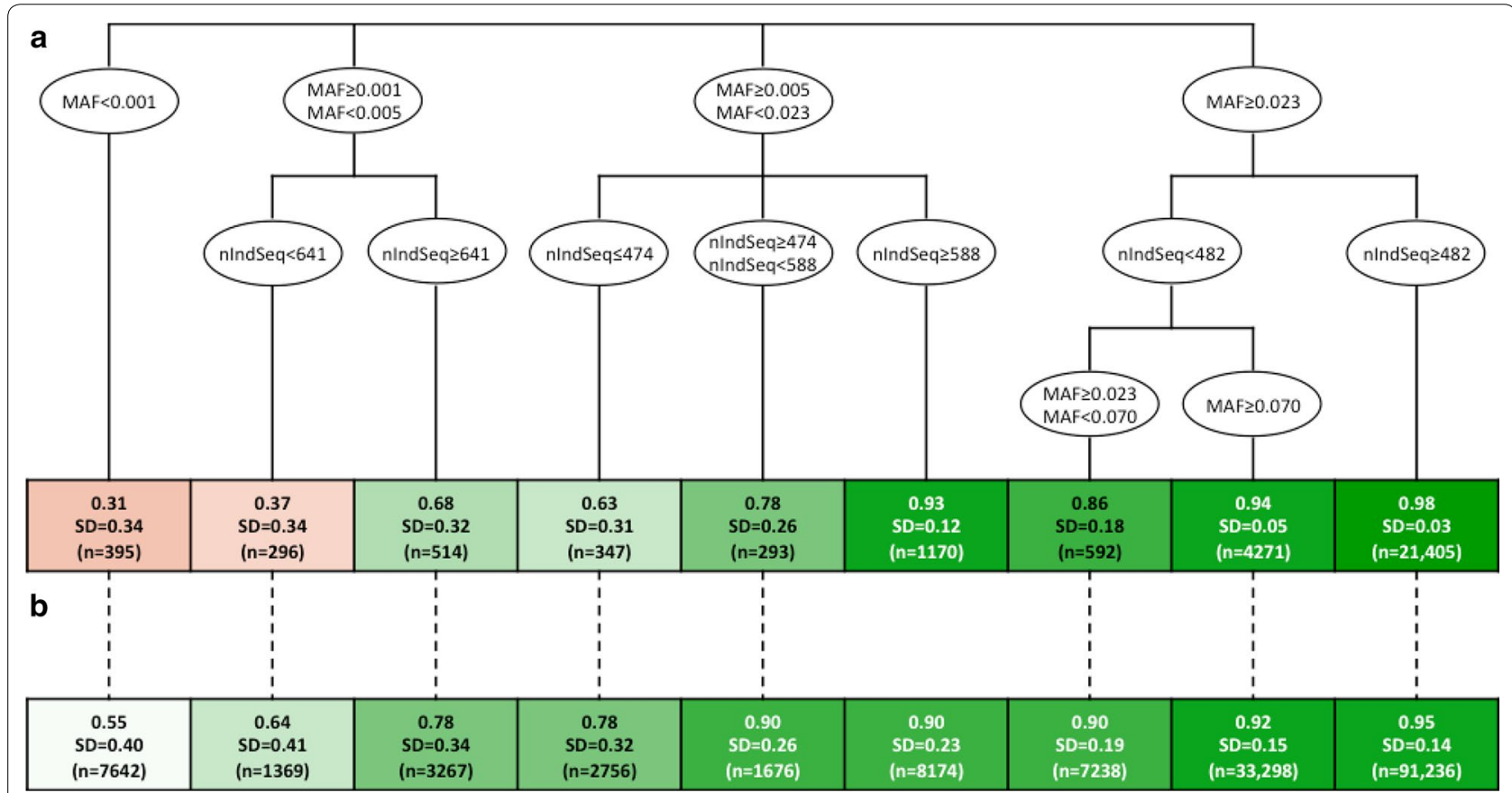

Fig. 4 Regression tree of the factors that affected variant-wise dosage correlation in $\mathbf{a}$ simulated data and $\mathbf{b}$ comparison to real data. Variables include minor allele frequency (MAF) and number of individuals sequenced at a position (nIndSeq)

\section{Impact of data misassignment and pedigree errors}

Data misassignment and pedigree errors can have drastic consequences on the imputation results. The impact of data misassignment and pedigree errors, measured as the dosage correlation between the results with and without the deliberate error, is presented in Fig. 5 for the target individual ('ind') and its immediate relatives. We report here the average dosage correlation but note that there was large case-by-case variability due to the stochasticity of the data misassignment and pedigree errors.

When we removed the high-coverage sequence data of the target individual, as in Test 1 (Fig. 5a), the dosage correlation with complete data imputation was 0.94 for the target individual. The impact of removing the sequence data of the target individual had a limited impact on imputing its relatives, which had dosage correlations of 0.97 to 0.99 compared to the case with complete data. 
a Sequence data masking

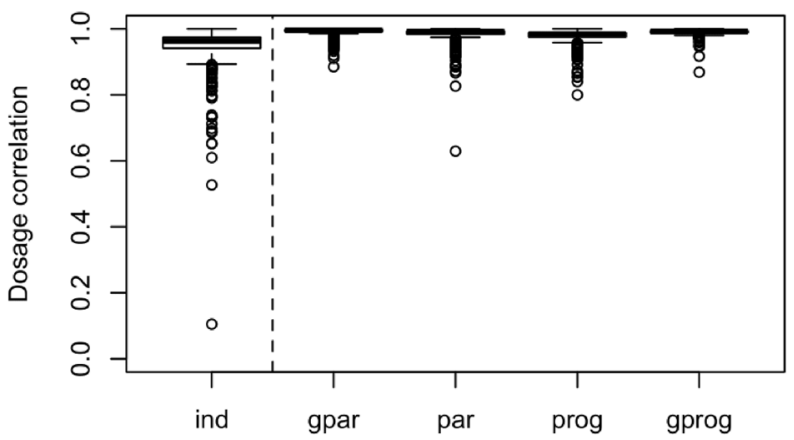

Relationship to target individual

c Marker array data misassignment

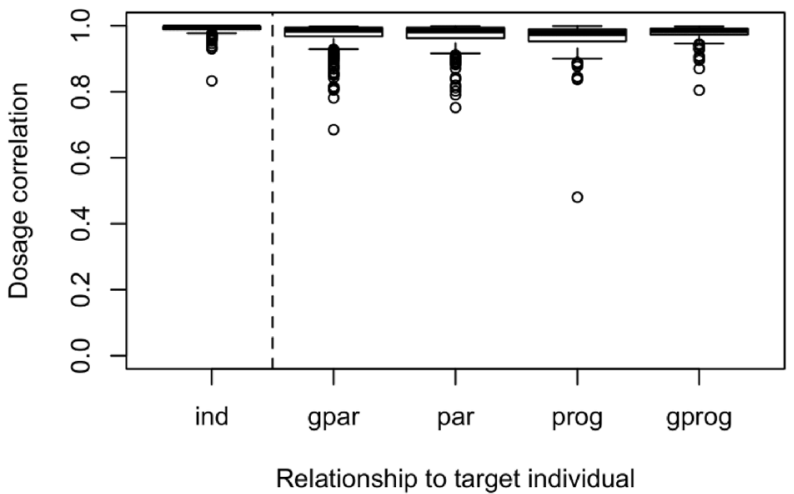

b Sequence data misassignment

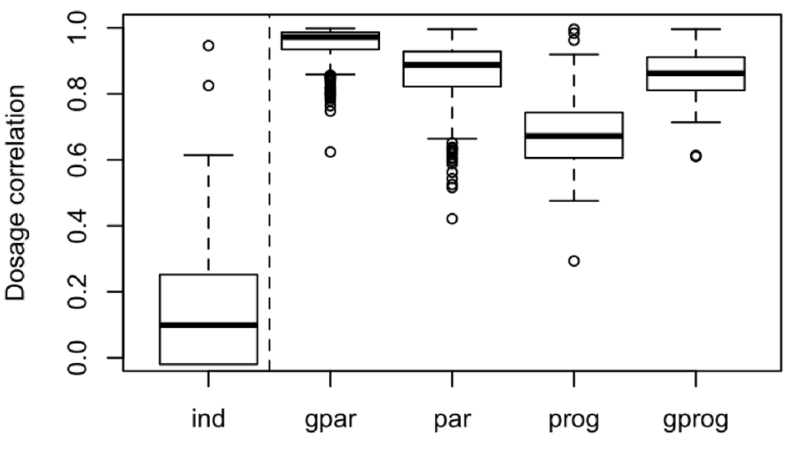

Relationship to target individual

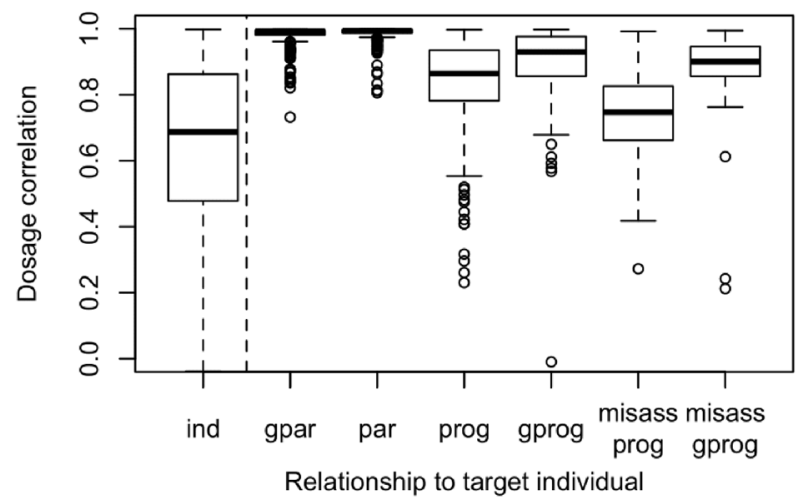

Fig. 5 Impact of data misassignment and pedigree errors on imputation accuracy. The dashed line separates the individual directly affected by the data modification (ind) and its relatives (gpar: grandparents, par: parents, prog: progeny, gprog: grandprogeny, misass prog: misassigned progeny, misass gprog: misassigned grandprogeny). The $y$-axis measures the individual-wise dosage correlation between the imputed genotypes based on complete correct data and either missing or misassigned data for the individual itself and its relatives. In panel a we provide the case where the sequence data of the target individual was masked as in Test 1 ; in panel $\mathbf{b}$ where the sequence data of another individual was misassigned to the target one; in panel $\mathbf{c}$ where the marker array data was misassigned; and in panel $\mathbf{d}$ where we assigned the progeny from one of the individuals sequenced at high coverage to the target individual

When the sequence data was misassigned (Fig. 5b), the dosage correlation of the target individual drastically decreased to 0.13 , as did (in order of magnitude) that of its progeny (0.68), then its grandprogeny $(0.86)$ and parents (0.86), and finally its grandparents (0.95).

When the marker array data was misassigned (Fig. 5c), the dosage correlation of the target individual remained very high (0.99), probably because the high-coverage sequence data provided high certainty about its true genotypes. In spite of this, potential errors in the segregation probabilities resulted in dosage correlations for the relatives of the target individual that were slightly lower $(0.97$ to 0.98 ) and showed a greater dispersion.
Finally, when the pedigree was misassigned (Fig. 5d), the impact of such errors depended on the number of true and misassigned relatives that the target individual had. In our test, the target individual was misassigned progeny from one of the individuals sequenced at high coverage. The dosage correlation of the target individual greatly decreased (0.65). The greatest impact of the pedigree errors was on the misassigned progeny (0.74), but the impact on the true progeny was also large $(0.83)$. The impact was smaller on the misassigned grandprogeny (0.89) and the true grandprogeny (0.90). The dosage correlation of the parents and grandparents of the target individual were largely unchanged $(0.99$ and 0.98 , respectively), probably because they had other correctly 
assigned relatives (like their own parents) that contributed more accurate data.

\section{Discussion}

In this paper, we present the results of a large-scale sequencing study that aimed at generating accurately imputed whole-genome sequence information on hundreds of thousands of individuals. Our results show that we were able to obtain highly accurate sequence information for approximately 230,000 individuals from four different populations that were genotyped at a maximum of 75,000 markers genome-wide, by sequencing only $2 \%$ of the individuals in each population, mostly at low coverage. We found that imputation accuracy was high for most individuals, especially for descendants of the first few generations of a pedigree. The same approach was applied to five additional populations (results not shown), providing high-quality whole-genome sequence data for more than 350,000 individuals. To our knowledge, this is the largest set of whole-genome sequence information assembled to date in pigs [33] or in any other livestock species (e.g., [7, 34]).

Our results give rise to five major points of discussion: (i) the overall performance of the sequencing strategy and the approach that we used for imputing whole-genome sequence data; (ii) the individual-wise imputation accuracy; (iii) the variant-wise imputation accuracy; (iv) the comparison to other imputation methods; and (v) the implications for population-wide sequencing studies.

\section{Overall performance of the sequencing strategy and hybrid peeling}

The overall performance of our sequencing strategy coupled with hybrid peeling was high. We were able to impute whole-genome sequence data for hundreds of thousands of individuals with a median dosage correlation of 0.97 by sequencing only about $2 \%$ of the individuals in each of our pedigreed populations. Most of the sequenced individuals were sequenced at low coverage, with $90 \%$ of the sequenced individuals at either $1 \times$ or $2 \times$ and only $6.4 \%$ of the sequenced individuals being sequenced at a high coverage of $15 \times$ to $30 \times$. Sequencing a subset of individuals at high coverage may improve the variant discovery rates as well as provide a validation set for variants discovered with low-coverage sequence data. It is difficult to separate the contributions of the sequencing strategy and of the imputation method to the imputation accuracy. We have assessed the contribution of the sequencing strategy on imputation accuracy in a companion paper [27]. Overall, sequencing coverage does not seem a very influential factor if a sufficiently large number of individuals is sequenced and, therefore, the sequencing strategy based primarily on low-coverage sequencing that we have described enabled high imputation accuracy in real livestock populations regardless of the size of the population.

Our sequencing strategy and imputation method enabled high imputation accuracy of whole-genome sequence data from marker arrays with relatively low densities, of approximately 15,000 and 75,000 markers genome-wide. The low dependence on marker arrays with higher densities contrasts with the findings of previous studies on imputation of whole-genome sequence data, which reported that marker array genotyping density was critical when using other sequencing strategies and imputation methods. For example, van Binsbergen et al. [35] found that imputing from marker arrays with a density similar to ours (50,000 markers genome-wide) resulted in low accuracies (dosage correlations of up to 0.80 ) when using the Beagle imputation software (version 3; [36]) in cattle. Van den Berg et al. [33] found similarly low accuracies in pigs (dosage correlations of $\sim 0.70$ ), probably because the number of sequenced individuals was small. In order to achieve higher imputation accuracies, an intermediate step of imputation to a much higher density (700,000 markers genome-wide or similar) was previously proposed [35]. This intermediate step has been used in several studies and with other imputation methods [33, 34, 37, 38], but this may be a drawback for populations where marker array data at such high densities are not available. We found that a combination of an appropriate sequencing strategy and hybrid peeling achieved high imputation accuracies without any intermediate imputation steps being required for the LD individuals. This was likely due to the ability of both methods to exploit pedigree and existing marker array information to maximise the value of the generated whole-genome sequence data for the whole population.

\section{Individual-wise imputation accuracy}

Although most of the individuals had high imputation accuracy, a small portion of individuals had much lower imputation accuracies than the rest. These individuals mostly belonged to the earliest generations of each pedigree. This reduction of imputation accuracy in the earliest generations of the pedigree was consistent with observations in previous simulation studies $[15,27]$. The individuals in these generations tend to have very little information available for themselves and for their ancestors, i.e., many of these individuals were not genotyped with marker arrays or their parents and grandparents were not genotyped either. Availability of marker array data from ancestors is critical for phasing and the accurate estimation of the segregation probabilities in the multi-locus step of hybrid peeling and it greatly affects the resulting accuracy of imputation accuracy. 
In a similar way, the marker array density at which the ancestors were genotyped affected imputation accuracy of an individual, regardless of the marker array density at which the individual itself was genotyped. This can be explained by the fact that parental and grandparental genotypes are needed for accurately phasing the individual's genotype and even a small number of markers is sufficient to capture the small number of recombinations between the individual and its parents [16]. Thus, strategies that target parents that contribute large numbers of progeny for genotyping at high density, such as current genotyping practices of breeding programs with genomic selection [39, 40], seem appropriate.

Provided that the segregation probabilities were accurately estimated, high connectedness of an individual to the rest of the population enhanced its imputation accuracy by favouring the transmission of information from many relatives and by increasing the likelihood that a closely connected individual has sequence data. In livestock breeding populations, it is usual that pedigrees are deep and individuals have a high degree of relatedness. The connectedness of the imputed individuals to a sufficient number of informative relatives with marker array or sequence data allows for high imputation accuracy (after the initial generations) even when only a small subset of individuals was sequenced at low levels of coverage.

It is possible to predict individuals with low imputation accuracy based on the availability of data for themselves and their relatives. This approach works especially well for filtering out those individuals with the lowest imputation accuracies as a first conservative data quality control step before downstream analyses. However, it is critical to perform quality controls of the data also before performing imputation to avoid any data misassignment or pedigree errors. In this study, we attempted to set an upper threshold for the impact that these errors could have on the individual-wise imputation accuracy of the affected individuals as well as how these errors propagate to the relatives of the affected individuals in a pedigree-based method. We found that the most serious errors occurred due to pedigree errors or assigning sequence data to a wrong individual. However, this may be distorted by the fact that all the target individuals had high-coverage sequence data. Therefore, misassignment of marker array data must not be ignored as it could also have a strong impact on imputation accuracy when it affects individuals that are not sequenced, sequenced at low coverage, or whose relatives are genotyped with lowdensity marker arrays. Fortunately, frameworks to detect data misassignment [41] and pedigree errors [42] have been developed and we have described an approach to correct such errors with little disruption of the pedigree structure. We did not test the impact that map errors could have on the imputation accuracy, but it is obvious that they would hamper the estimation of the segregation probabilities and thus imputation accuracy.

\section{Variant-wise imputation accuracy}

We obtained high variant-wise imputation accuracy after filtering out individuals that were predicted to have low imputation accuracy. The primary factor for variant-wise imputation accuracy was MAF. This was expected, as MAF is widely known to be one of the main factors that determine imputation accuracy regardless of the imputation method, and we found, similar to other studies, that imputation accuracy was lower for variants with very low MAF $[4,35,37,43]$.

The next most important factor was the number of individuals that had sequence data that variant site. Low-coverage sequencing results in a sparse distribution of reads along the genome, and it is likely that only a subset of the sequenced individuals will have any reads that map to a given variant site and that the cumulative coverage across variant sites will also vary. In our study, the number of individuals with some coverage and the cumulative coverage may be confounded because most individuals were sequenced at $1 \times$ or $2 \times$, but in general this indicates the importance of having as many sequenced individuals as possible with some coverage at each variant site [27], a situation that is favoured by sequencing strategies based on low coverage.

The importance of the number of individuals that had sequence data at a variant site also suggests that imputation accuracy could be lower in regions with extreme base compositions or particular sequence motifs that hamper read alignment $[44,45]$. While the complexity of a given region, namely the presence of large repeats, is another factor that could affect local imputation accuracy along a chromosome $[37,46]$, it was not considered in our study.

Inferring the segregation probabilities from the flanking markers that are included in the marker array did not result in noticeably lower imputation accuracy for those variants that were not included in the marker array. Moreover, variant-wise imputation accuracy was found to be independent of the distance between the variant and the flanking markers at which the segregation probabilities were estimated. These findings differed from those of previous studies using methods based on linkage disequilibrium (Beagle, version 3; [36]), where variant-wise imputation accuracy decreased as the distance between each variant and the nearest variant in the marker array (from which imputation to whole-genome sequence data was performed) increased [35]. 


\section{Comparison to other imputation methods}

We did not intend to make a direct comparison of the performance of hybrid peeling with other available imputation methods because there are fundamental differences in how they exploit information (pedigree and linkage vs. linkage disequilibrium) and because sequencing strategies and imputation methods are confounded across studies. However, we have previously compared the performance of our hybrid peeling with findhap (version 4; [43]) [15] and other studies have compared other available imputation tools [37, 38, 43, 47], including tools such as Beagle (versions 3 and 4; [36, 48]), IMPUTE2 [49], findhap [43], FImpute [50], or Minimac3 [51]. Many of these methods are population-based imputation methods that use an already phased haplotype reference panel to impute genotyped individuals to whole-genome sequence data. As a consequence, previous studies of the factors that influence imputation accuracy have been primarily concerned with the design of the reference panel. Some of these concerns involve the convenience of using single-breed or multi-breed reference panels $[38,47]$, population-specific reference panels [38, 52], the availability of marker array data for the sequenced individuals or not (it removes the genotype uncertainty that otherwise would arise from sequencing at low coverage at some pre-established positions) [43], or the trade-off between number of individuals sequenced and sequencing coverage [43]. In contrast, in this paper, we used a purely pedigree-based imputation algorithm. This allows us to exploit the large amount of linkage between the haplotypes of an individual and their relatives.

\section{Implications for population-wide sequencing studies}

The coupling of an appropriate sequencing strategy [13, 14, 27] and an appropriate imputation method, such as hybrid peeling [15], enabled the generation of large datasets of sequenced individuals at a low cost and with high accuracy. This is a critical step for the successful implementation of whole-genome sequence data for genomic prediction, within and across breeds, as well as for finemapping of causal variants underlying quantitative traits, which could guide the promotion and removal of alleles by gene editing $[53,54]$.

In this paper, we focused on individual-wise imputation accuracy as an indicator of the value of this data for applications such as genomic prediction. Previous studies on imputation accuracy of whole-genome sequence data focused on variant-wise imputation accuracy rather than individual-wise $[35,37,43]$. In the context of genomic prediction, the estimate of the realized relationship between two individuals will correlate strongly with the individual-wise, but not the variant-wise, imputation accuracy $[31,55]$. Understanding which factors determine the variability of individual-wise, as well as variant-wise [35, 37], imputation accuracy would enable accuracy-aware filtering of the imputed data prior to downstream analyses. With that purpose, we used regression trees on simulated data designed to mimic the real data for identifying a small set of partitioning factors that may be used as predictors to filter out individuals with expected low imputation accuracy.

\section{Conclusions}

We demonstrate the high accuracy of hybrid peeling for imputing whole-genome sequence data of hundreds of thousands of individuals from real livestock populations in which only a small fraction of the individuals (2\%) had been sequenced, mostly at low coverage. Using data from pig populations, we show that imputation accuracy was very high for individuals that were genotyped with marker arrays with densities that ranged between 15,000 and 75,000 markers genome-wide. The coupling of an appropriate sequencing strategy and hybrid peeling is a powerful method for generating whole-genome sequence data in large pedigreed populations, as long as the individuals are connected to enough informative relatives with marker array or sequence data, and regardless of population size. The characterization of the factors that affect the individual-wise and variantwise imputation accuracy of hybrid peeling can inform genotyping and sequencing strategies as well as provide accuracy-aware quality control guidelines for the imputed data before downstream analyses. The success of this sequencing strategy demonstrates the possibility of obtaining low-cost whole-genome sequence data on large pedigreed livestock populations, which is a critical step for the successful implementation of wholegenome sequence data for genomic prediction and fine-mapping of causal variants.

\section{Acknowledgements \\ This work has made use of the resources provided by the Edinburgh Compute and Data Facility (ECDF) (http://www.ecdf.ed.ac.uk/).}

\section{Authors' contributions}

RRF, AW, and JMH designed the study; RRF and CYC performed the analyses; RRF wrote the first draft; AW, GG, WOH, AJM, and JMH assisted in the interpretation of the results and provided comments on the manuscript. All authors read and approved the final manuscript.

\section{Funding}

The authors acknowledge the financial support from the BBSRC ISPG to The Roslin Institute (BBS/E/D/30002275), from Genus plc, Innovate UK (Grant 102271), and from Grant numbers BB/N004736/1, BB/N015339/1, BB/ L020467/1, and BB/M009254/1.

\section{Availability of data and materials}

The software packages AlphaSim, AlphaSeqOpt, AlphaPhase, Alphalmpute and AlphaPeel are available from the AlphaGenes website (http://www.alpha genes.roslin.ed.ac.uk). The datasets generated and analysed in this study are derived from the PIC breeding programme and not publicly available. 


\section{Ethics approval and consent to participate}

The samples used in this study were derived from the routine breeding activities of PIC.

\section{Consent for publication}

Not applicable.

\section{Competing interests}

The authors declare that they have no competing interests.

\section{Author details}

${ }^{1}$ The Roslin Institute and Royal (Dick) School of Veterinary Studies, The University of Edinburgh, Easter Bush, Midlothian, Scotland, UK. ${ }^{2}$ Departament de Ciència Animal, Universitat de Lleida-Agrotecnio Center, Lleida, Spain. ${ }^{3}$ The Pig Improvement Company, Genus plc, 100 Bluegrass Commons Blvd Ste 2200, Hendersonville, TN 37075, USA. ${ }^{4}$ Genus plc, 1525 River Road, Deforest, WI 53532, USA.

Received: 16 September 2019 Accepted: 27 March 2020

Published online: 06 April 2020

\section{References}

1. Pasaniuc B, Rohland N, McLaren PJ, Garimella K, Zaitlen N, Li H, et al. Extremely low-coverage sequencing and imputation increases power for genome-wide association studies. Nat Genet. 2012;44:631-5.

2. Daetwyler HD, Capitan A, Pausch H, Stothard P, van Binsbergen R, Brondum RF, et al. Whole-genome sequencing of 234 bulls facilitates mapping of monogenic and complex traits in cattle. Nat Genet. 2014:46:858-65.

3. Nicod J, Davies RW, Cai N, Hassett C, Goodstadt L, Cosgrove C, et al. Genome-wide association of multiple complex traits in outbred mice by ultra-low-coverage sequencing. Nat Genet. 2016;48:912-8.

4. Sanchez MP, Govignon-Gion A, Croiseau P, Fritz S, Hozé C, Miranda G, et al. Within-breed and multi-breed GWAS on imputed whole-genome sequence variants reveal candidate mutations affecting milk protein composition in dairy cattle. Genet Sel Evol. 2017;49:68.

5. Meuwissen T, Goddard M. Accurate prediction of genetic values for complex traits by whole-genome resequencing. Genetics. 2010;185:623-31.

6. Druet T, Macleod IM, Hayes BJ. Toward genomic prediction from wholegenome sequence data: impact of sequencing design on genotype imputation and accuracy of predictions. Heredity. 2014;112:39-47.

7. Raymond B, Bouwman AC, Schrooten C, Houwing-Duistermaat J, Veerkamp RF. Utility of whole-genome sequence data for across-breed genomic prediction. Genet Sel Evol. 2018;50:27.

8. Das A, Panitz F, Gregersen VR, Bendixen C, Holm L-E. Deep sequencing of Danish Holstein dairy cattle for variant detection and insight into potential loss-of-function variants in protein coding genes. BMC Genomics. 2015:16:1043.

9. Gudbjartsson DF, Helgason H, Gudjonsson SA, Zink F, Oddson A, Gylfason $A$, et al. Large-scale whole-genome sequencing of the Icelandic population. Nat Genet. 2015;47:435-44.

10. Li Y, Sidore C, Kang HM, Boehnke M, Abecasis GR. Low-coverage sequencing: implications for design of complex trait association studies. Genome Res. 2011;21:940-51.

11. Hickey JM. Sequencing millions of animals for genomic selection 2.0. J Anim Breed Genet. 2013;130:331-2.

12. Hickey JM, Gorjanc G, Cleveland MA, Kranis A, Jenko J, Mésázros G, et al. Sequencing millions of animals for Genomic Selection 2.0. In Proceedings of the 10th World Congress on Genetics Applied to Livestock Production: 17-22 August 2014; Vancouver; 2014. p. 377.

13. Gonen S, Ros-Freixedes R, Battagin M, Gorjanc G, Hickey JM. A method for the allocation of sequencing resources in genotyped livestock populations. Genet Sel Evol. 2017;49:47.

14. Ros-Freixedes R, Gonen S, Gorjanc G, Hickey JM. A method for allocating low-coverage sequencing resources by targeting haplotypes rather than individuals. Genet Sel Evol. 2017;49:78.

15. Whalen A, Ros-Freixedes R, Wilson DL, Gorjanc G, Hickey JM. Hybrid peeling for fast and accurate calling, phasing, and imputation with sequence data of any coverage in pedigrees. Genet Sel Evol. 2018;50:67.
16. Hickey JM, Kinghorn BP, Tier B, Wilson JF, Dunstan N, van der Werf JH. A combined long-range phasing and long haplotype imputation method to impute phase for SNP genotypes. Genet Sel Evol. 2011;43:12.

17. Hickey JM, Kinghorn BP, Tier B, van der Werf JH, Cleveland MA. A phasing and imputation method for pedigreed populations that results in a single-stage genomic evaluation. Genet Sel Evol. 2012;44:9.

18. Bolger AM, Lohse M, Usadel B. Trimmomatic: a flexible trimmer for illumina sequence data. Bioinformatics. 2014;30:2114-20.

19. Warr A, Affara N, Aken B, Beiki H, Bickhart DM, Billis K, et al. An improved pig reference genome sequence to enable pig genetics and genomics research. BioRxiv. 2019. https://doi.org/10.1101/668921.

20. Li H. Aligning sequence reads, clone sequences and assembly contigs with BWA-MEM. ArXiv. 2013;1303.3997v1 [q - bio.GN].

21. DePristo MA, Banks E, Poplin R, Garimella KV, Maguire JR, Hartl C, et al. A framework for variation discovery and genotyping using next-generation DNA sequencing data. Nat Genet. 2011;43:491-8.

22. Poplin R, Ruano-Rubio V, DePristo MA, Fennell TJ, Carneiro MO, Van der Auwera GA, et al. Scaling accurate genetic variant discovery to tens of thousands of samples. BioRxiv. 2018. https://doi.org/10.1101/201178.

23. Ros-Freixedes R, Battagin M, Johnsson M, Gorjanc G, Mileham AJ, Rounsley SD, et al. Impact of index hopping and bias towards the reference allele on accuracy of genotype calls from low-coverage sequencing. Genet Sel Evol. 2018;50:64.

24. Li H, Handsaker B, Wysoker A, Fennell T, Ruan J, Homer N, et al. The sequence alignment/map format and SAMtools. Bioinformatics. 2009;25:2078-9.

25. Danecek P, Auton A, Abecasis G, Albers CA, Banks E, DePristo MA, et al. The variant call format and VCFtools. Bioinformatics. 2011;27:2156-8.

26. Faux A-M, Gorjanc G, Gaynor RC, Battagin M, Edwards SM, Wilson DL, et al. AlphaSim: software for breeding program simulation. Plant Genome. 2016. https://doi.org/10.3835/plantgenome2016.02.0013.

27. Ros-Freixedes R, Whalen A, Gorjanc G, Mileham AJ, Hickey JM. Evaluation of sequencing strategies for whole-genome imputation with hybrid peeling. Genet Sel Evol. 2020. https://doi.org/10.1186/s12711-020-00537 $-7$

28. Gorjanc G, Dumasy J-F, Gonen S, Gaynor RC, Antolin R, Hickey JM Potential of low-coverage genotyping-by-sequencing and imputation for cost-effective genomic selection in biparental segregating populations. Crop Sci. 2017;57:1404-20.

29. Kerr RJ, Kinghorn BP. An efficient algorithm for segregation analysis in large populations. J Anim Breed Genet. 1996;113:457-69.

30. Meuwissen T, Goddard M. The use of family relationships and linkage disequilibrium to impute phase and missing genotypes in up to wholegenome sequence density genotypic data. Genetics. 2010;185:1441-9.

31. Calus MPL, Bouwman AC, Hickey JM, Veerkamp RF, Mulder HA. Evaluation of measures of correctness of genotype imputation in the context of genomic prediction: a review of livestock applications. Animal. 2014;8:1743-53.

32. Therneau T, Atkinson B, Ripley B. Rpart: recursive partitioning and regression trees (R package version 4.1-11). 2017. https://CRAN.R-project.org/ package=rpart Accessed 13 March 2020.

33. van den Berg S, Vandenplas J, van Eeuwijk FA, Bouwman AC, Lopes MS, Veerkamp RF. Imputation to whole-genome sequence using multiple pig populations and its use in genome-wide association studies. Genet Sel Evol. 2019;51:2.

34. Ring SC, Purfield DC, Good M, Breslin P, Ryan E, Blom A, et al. Variance components for bovine tuberculosis infection and multi-breed genomewide association analysis using imputed whole genome sequence data. PLoS One. 2019;14:e0212067.

35. van Binsbergen R, Bink MC, Calus MP, van Eeuwijk FA, Hayes BJ, Hulsegge I, et al. Accuracy of imputation to whole-genome sequence data in Holstein Friesian cattle. Genet Sel Evol. 2014;46:41.

36. Browning BL, Browning SR. A unified approach to genotype imputation and haplotype-phase inference for large data sets of trios and unrelated individuals. Am J Hum Genet. 2009;84:210-23.

37. Bolormaa S, Chamberlain AJ, Khansefid M, Stothard P, Swan AA, Mason $B$, et al. Accuracy of imputation to whole-genome sequence in sheep. Genet Sel Evol. 2019;51:1.

38. Korkuć P, Arends D, Brockmann GA. Finding the optimal imputation strategy for small cattle populations. Front Genet. 2019;10:52. 
39. Huang Y, Hickey JM, Cleveland MA, Maltecca C. Assessment of alternative genotyping strategies to maximize imputation accuracy at minimal cost. Genet Sel Evol. 2012;44:25.

40. Cleveland MA, Hickey JM. Practical implementation of cost-effective genomic selection in commercial pig breeding using imputation. J Anim Sci. 2013;91:3583-92.

41. Chan AW, Williams AL, Jannink JL. A statistical framework for detecting mislabeled and contaminated samples using shallow-depth sequence data. BMC Bioinformatics. 2018:19:478.

42. Whalen A, Gorjanc G, Hickey JM. Parentage assignment with genotypingby-sequencing data. J Anim Breed Genet. 2018;136:102-12.

43. VanRaden PM, Sun C, O'Connell JR. Fast imputation using medium or low-coverage sequence data. BMC Genet. 2015;16:82.

44. Ross MG, Russ C, Costello M, Hollinger A, Lennon NJ, Hegarty R, et al. Characterizing and measuring bias in sequence data. Genome Biol. 2013;14:R51.

45. Brandt DYC, Aguiar VRC, Bitarello BD, Nunes K, Goudet J, Meyer D. Mapping bias overestimates reference allele frequencies at the HLA genes in the 1000 genomes project phase I data. G3 (Bethesda). 2015;5:931-41.

46. Pausch H, MacLeod IM, Fries R, Emmerling R, Bowman PJ, Daetwyler HD, et al. Evaluation of the accuracy of imputed sequence variant genotypes and their utility for causal variant detection in cattle. Genet Sel Evol. 2017;49:24.

47. Brøndum R, Guldbrandtsen B, Sahana G, Lund M, Su G. Strategies for imputation to whole genome sequence using a single or multi-breed reference population in cattle. BMC Genomics. 2014;15:728.

48. Browning BL, Browning SR. Genotype imputation with millions of reference samples. Am J Hum Genet. 2016;98:116-26.
49. Howie BN, Donnelly P, Marchini J. A flexible and accurate genotype imputation method for the next generation of genome-wide association studies. PLoS Genet. 2009;5:e1000529.

50. Sargolzaei M, Chesnais JP, Schenkel FS. A new approach for efficient genotype imputation using information from relatives. BMC Genomics. 2014; 15:478.

51. Das S, Forer L, Schönherr S, Sidore C, Locke AE, Kwong A, et al. Nextgeneration genotype imputation service and methods. Nat Genet. 2016;48:1284-7.

52. Lencz T, Yu J, Palmer C, Carmi S, Ben-Avraham D, Barzilai N, et al. Highdepth whole genome sequencing of an Ashkenazi Jewish reference panel: enhancing sensitivity, accuracy, and imputation. Hum Genet. 2018;137:343-55

53. Jenko J, Gorjanc G, Cleveland MA, Varshney RK, Whitelaw CBA, Woolliams $J A$, et al. Potential of promotion of alleles by genome editing to improve quantitative traits in livestock breeding programs. Genet Sel Evol. 2015;47:55

54. Johnsson M, Gaynor RC, Jenko J, Gorjanc G, de Koning DJ, Hickey JM. Removal of alleles by genome editing (RAGE) against deleterious load. Genet Sel Evol. 2019;51:14.

55. Whalen A, Gorjanc G, Hickey JM. Family-specific genotype arrays increase the accuracy of pedigree-based imputation at very low marker densities. Genet Sel Evol. 2019;51:33.

\section{Publisher's Note}

Springer Nature remains neutral with regard to jurisdictional claims in published maps and institutional affiliations.
Ready to submit your research? Choose BMC and benefit from:

- fast, convenient online submission

- thorough peer review by experienced researchers in your field

- rapid publication on acceptance

- support for research data, including large and complex data types

- gold Open Access which fosters wider collaboration and increased citations

- maximum visibility for your research: over $100 \mathrm{M}$ website views per year

At BMC, research is always in progress.

Learn more biomedcentral.com/submissions 